\title{
A German Study Comparing Standard Wire Localization With Magnetic Seed Localization of Non-palpable Breast Lesions
}

\author{
FRIEDRICH KÜHN $^{1 *}$, CHARLOTTE EMMI ELISABETH SIMON ${ }^{2 *}$, ILHAMIYYA ALIYEVA ${ }^{1}$, \\ JULIA KUBMAUL ${ }^{1}$, JESSICA GROß ${ }^{1}$, OLIVER SCHWEIZERHOF ${ }^{3,4}$, \\ JENS-UWE BLOHMER $^{1}$ and MARIA MARGARETE KARSTEN ${ }^{1}$ \\ ${ }^{1}$ Charité - Universitätsmedizin Berlin, Corporate Member of Freie Universität Berlin, \\ Humboldt-Universität zu Berlin and Berlin Institute of Health, \\ Clinic for Gynecology with Breast Center, Berlin, Germany; \\ ${ }^{2}$ Department of Obstetrics and Gynecology, University Hospital, LMU Munich, Munich, Germany; \\ ${ }^{3}$ Charité - Universitätsmedizin Berlin, Corporate Member of Freie Universität Berlin, Humboldt-Universität zu Berlin, \\ and Berlin Institute of Health, Institute of Biometry and Clinical Epidemiology, Berlin, Germany; \\ ${ }^{4}$ Berlin Institute of Health (BIH), Berlin, Germany
}

\begin{abstract}
Background: Exact localization of non-palpable breast lesions is necessary to ensure that the correct lesion is removed. Conventional methods come with several disadvantages. Patients and Methods: We compared 28 patients who underwent breast-conserving surgery for a nonpalpable lesion. By surgeon choice, 14 patients were assigned to undergo magnetic seed localization and 14 underwent standard wire localization. The primary outcome was the operative time, and secondary outcome was the patient pain level. Results: The mean age was $52 \pm 10$ (SD) years in the seed arm, and $55 \pm 13$ years in the wire arm. The median time from skin incision to tumor extraction was not significantly different between the two groups. Patients in the wire localized group significantly more often reported pain during coughing/breathing, movement, and sleep. Conclusion: Using seed localization at Charité Breast Center did not lead to a significant decrease in operative time but might allow time savings once established, while increasing patient comfort and reducing organizational burden.
\end{abstract}

This article is freely accessible online.

*FK and CEES are shared first Authors.

Correspondence to: Maria Margarete Karsten, Charité Universitätsmedizin Berlin, Klinik für Gynäkologie mit Brustzentrum, Charitéplatz 1, 10117 Berlin, Germany. Tel: +49 30450664825, e-mail: maria-margarete.karsten@charite.de

Key Words: Breast cancer, magnetic seeds, wire localization, breast conserving therapy, non-palpable breast lesions.
The number of diagnosed non-palpable breast lesions is increasing due to well-established breast cancer screening methods and improved imaging technologies (1). It is one of the main goals in breast surgery to preserve as much healthy tissue as possible while gaining tumor-free margins, avoiding the necessity of re-intervention and achieving an oncologically safe and cosmetically satisfactory outcome.

In order to reach this goal, it is necessary to mark the exact localization of the lesion prior to surgery. In most European countries wire-guided localization (WGL) is used for this purpose. Guided by imaging technologies such as mammography, ultrasound or magnetic resonance imaging, a wire is placed in the center of the lesion. There are certain well-described disadvantages of WGL, such as difficulty in placing the wire precisely, its migration or dislocation, as well as the surgical approach being limited by the entry point of the wire. It can also cause patient discomfort or pain and logistical problems due to the fact that the wire must be placed on the day of the operation, which can result in inconvenience and inefficiencies regarding surgery schedules $(2,3)$.

To overcome those disadvantages, certain alternative localization methods have been evaluated, such as intraoperative ultrasound-guided resection, radio-guided occult lesion localization and radioactive seed localization (RSL) (3). These methods have been shown to represent equivalent or partly even superior alternatives to WGL (2, 4, $5)$. Yet they are associated with adverse requirements: In the case of intraoperative ultrasound-guided resection, the lesion must be visible on ultrasound. In addition, this technique requires certain training of the surgeon before it can be used successfully. When it comes to radio-guided methods, inconvenient radiation safety requirements must be taken into account. 


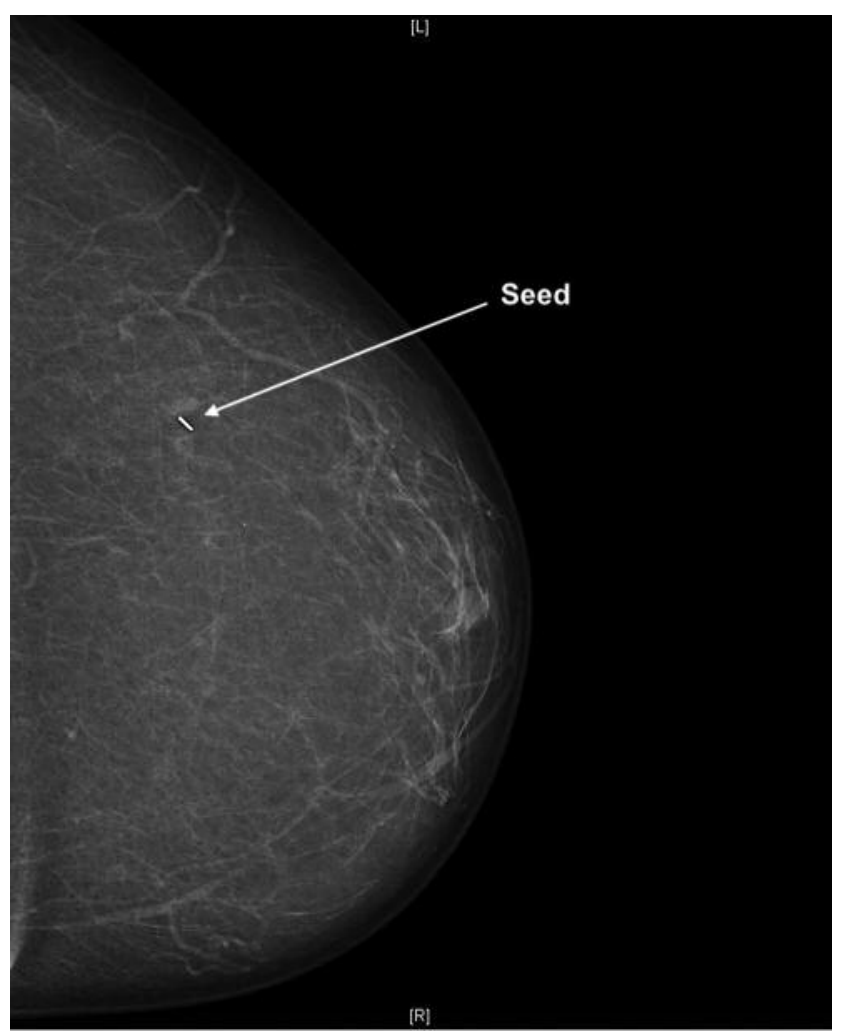

Figure 1. Mammography image after seed-localization of an impalpable breast tumor.

The use of magnetic seeds (Magseeds ${ }^{\circledR}$ ) is a relatively new method for localization of non-palpable breast lesions. Similarly to the use of radioactive seeds, it overcomes certain disadvantages of WGL while avoiding the difficulties associated with radioactivity.

Magseeds ${ }^{\circledR}$ (Endomagnetics Ltd., Cambridge, UK) (Figure 1) are small in size $(1 \times 5 \mathrm{~mm})$ and made of medical grade stainless steel. Application can be performed under ultrasound or radiography guidance using the supplied needle. The Sentimag ${ }^{\circledR}$ probe (Endomagnetics Ltd.) is then used to detect the seed during the operation. Exact application before and safe detection and retrieval during operation demonstrate the safety of their use even in large breasts (6). When compared to WGL, free margin rates are similar for both methods (7).

The aim of this study was to compare WGL with Magseed ${ }^{\circledR}$ localization regarding the psychological and physical discomfort of the patient and possible improvements in the organizational aspects at the Charité Breast Cancer Center.

\section{Patients and Methods}

After obtaining written consent, demographical, pre- and intraoperative data of 28 women requiring breast-conserving
Table I. General patient data and tumor characteristics.

\begin{tabular}{|c|c|c|c|}
\hline Characteristic & Seed $(n=14)$ & Wire $(n=14)$ & $p$-Value \\
\hline \multicolumn{4}{|l|}{ Age } \\
\hline Mean \pm SD & $51.9 \pm 10.1$ & $54.9 \pm 13.3$ & $0.508^{a}$ \\
\hline \multicolumn{4}{|l|}{ BMI $\left[\mathrm{kg} / \mathrm{m}^{2}\right]$} \\
\hline Median (IQR) & $23.6(20.2-30.0)$ & $23.3(21.3-32.0)$ & $0.894^{\mathrm{b}}$ \\
\hline$<25 \mathrm{~kg} / \mathrm{m}^{2}$ & $9(69.2 \%)$ & $7(58.3 \%)$ & \\
\hline$\geq 25 \mathrm{~kg} / \mathrm{m}^{2}$ & $4(30.8 \%)$ & $5(41.7 \%)$ & $0.688^{c}$ \\
\hline Missing & 1 & 2 & \\
\hline \multicolumn{4}{|l|}{$\begin{array}{l}\text { Microcalcifications } \\
(\mathrm{MMG}), \mathrm{n}(\%)\end{array}$} \\
\hline No & $11(84.6 \%)$ & $4(28.6 \%)$ & $0.006^{\mathrm{c}}$ \\
\hline Yes & $2(15.4 \%)$ & $10(71.4 \%)$ & \\
\hline Missing & 1 & 0 & \\
\hline \multicolumn{4}{|l|}{ Tumor size (US) [mm] } \\
\hline Median (IQR) & $8.5(6.8-10.3)$ & $11.0(5.0-16.0)$ & $0.467^{b}$ \\
\hline Missing & 0 & 3 & \\
\hline \multicolumn{4}{|l|}{ Tumor type, n (\%) } \\
\hline Benign & $3(21.4 \%)$ & $3(21.4 \%)$ & $>0.99^{\mathrm{c}}$ \\
\hline $\begin{array}{l}\text { Malignant/premalignant } \\
\text { (DCIS/LCIS) }\end{array}$ & $11(78.6 \%)$ & $11(78.6 \%)$ & \\
\hline \multicolumn{4}{|l|}{ Diagnosis, $\mathrm{n}(\%)$} \\
\hline Primary disease & $10(71.4 \%)$ & $8(57.1 \%)$ & $0.695^{\mathrm{c}}$ \\
\hline Recurrence & $4(28.6 \%)$ & $6(42.9 \%)$ & \\
\hline \multicolumn{4}{|l|}{ Resection margin, $\mathrm{n}(\%)$} \\
\hline R0 & $12(85.7 \%)$ & $10(71.4 \%)$ & $0.648^{\mathrm{c}}$ \\
\hline $\mathrm{R} 1$ & $2(14.3 \%)$ & $4(28.6 \%)$ & \\
\hline
\end{tabular}

\begin{tabular}{lccc}
$\begin{array}{l}\text { Subset of patients with } \\
\text { malignant tumor }\end{array}$ & & & \\
N (\%) & $\mathbf{1 1}(\mathbf{7 8 . 6 \%})$ & $\mathbf{1 1} \mathbf{( 7 8 . 6 \% )}$ & \\
$\begin{array}{l}\text { DCIS/accompanying } \\
\text { DCIS, n (\%) }\end{array}$ & & & \\
Yes & $6(54.5 \%)$ & $9(81.8 \%)$ & $0.361^{\mathrm{c}}$ \\
No & $5(45.5 \%)$ & $2(18.2 \%)$ & \\
Microcalcifications & & & \\
(MMG), n (\%) & & & \\
Yes & $2(20.0 \%)$ & $8(72.7 \%)$ & $0.030^{\mathrm{c}}$ \\
No & $8(80.0 \%)$ & $3(27.3 \%)$ & \\
Missing & 1 & 0 & \\
Tumor stage, n (\%) & & & \\
pTis & $0(0 \%)$ & $5(45.5 \%)$ & $0.018^{\mathrm{d}}$ \\
ypT0 & $1(9.1 \%)$ & $2(18.2 \%)$ & \\
pT1 & $10(90.9 \%)$ & $3(27.3 \%)$ & \\
pT2 & $0(0.0 \%)$ & $1(9.1 \%)$ & \\
\hline
\end{tabular}

SD: Standard deviation; IQR: interquartile range; DCIS: ductal carcinoma in situ; LCIS: lobular carcinoma in situ; MMG: mammography; R0/R1: negative resection margin/positive resection margin; US: ultrasound. ${ }^{\mathrm{a}} t$-Test for independent samples. ${ }^{\mathrm{b}}$ MannWhitney $U$-test. 'Fisher's exact test. ${ }^{\mathrm{d}} \mathrm{Chi}$-square test. Bold values refer to the subset of patients with malignant tumor.

surgery for a non-palpable lesion was collected between May 2018 and January 2019 in the breast center of Charité Universitätsmedizin Berlin. Exclusion criteria were palpable tumors and previous vacuum biopsy, as well as rejection of participation. Half of the cohort underwent tumor localization 


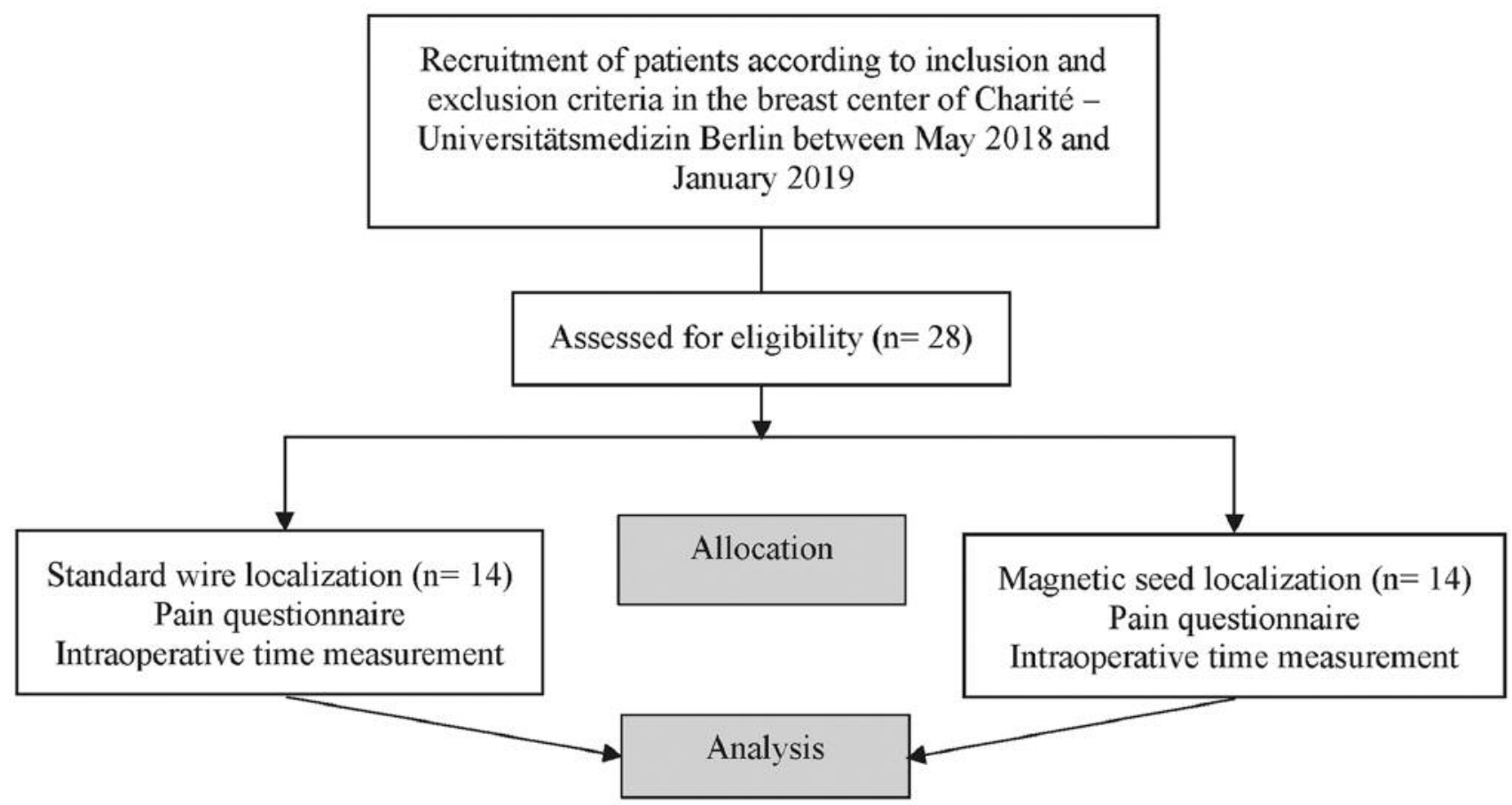

Figure 2. Consort diagram showing recruitment and group allocation for the study.

using magnetic seeds. For the other half, tumors were marked conventionally with wires. The group allocation was by surgeon choice. All of the included patients were asked to answer the quality improvement in postoperative pain management questionnaire (QUIPS) directly following the localization procedure. Among others, the questionnaire contains questions about pain levels, stress-related pain and resulting restriction of breathing/coughing, sleep and movement. Intraoperatively, time was measured between incision and tumor removal. Figure 2 illustrates the course of the study.

Besides general characteristics such as age and body mass index, clinical features including (sonographic) tumor size and presence of microcalcifications in the mammography were noted. We also differentiated between primary and recurrent disease and recorded the histological results (i.e. tumor type, tumor stage, resection margins).

Statistical analysis. Data collection was conducted in Excel (Office Excel 2016; Microsoft, Redmond, MA, USA) followed by statistical analyses using SPSS Statistics (SPSS Statistics Version 25; IBM, Armonk, NY, USA) and R statistical software (R Foundation for Statistical Computing, Vienna, Austria). Univariate comparisons between the seed and wire arm were made using Fisher's exact test or chi-square test for categorial variables. In the case of continuous variables, two-sample $t$-test or Mann-Whitney $U$-test was applied, depending on the data distribution. The significance level was set to alpha $=0.05$ without adjustment for multiple comparison. All $p$-values constitute exploratory data analysis.

\section{Results}

Patient cohort. All 28 patients were included in the analysis. Table I shows general patient data and tumor characteristics of both treatment groups. The mean age in the seed group was $52 \pm 10$ years and $55 \pm 13$ years in the wire group. There were no significant differences regarding tumor type and size. Eleven $(78.6 \%)$ patients in each group were diagnosed with malignant or premalignant tumors. Significantly more patients of the wire arm had mammographic microcalcifications compared to the seed arm (71.4\% versus $15.4 \%, p=0.006)$. This was similar in the subset of patients with (pre)malignant lesion. In this subset, there were slightly more patients diagnosed with a ductal carcinoma in situ (DCIS) or accompanying DCIS in the wire arm compared to the seed arm $(81.8 \%$ versus $54.5 \%, p=0.361)$. Concerning the tumor stage, all but one case within the seed arm were classified as pT1, whereas tumor stages varied more in the wire arm (Table I, $p=0.018$ ).

Resection time and pain levels. As shown in Figure 3, no significant difference in surgical time was identified, although the median resection time in the seed arm was slightly lower compared to the wire arm [14 (interquartile range=11-18) $\mathrm{min}$ vs. 17.5 (interquartile range $=11-24$ ) $\min , p=0.541]$. 


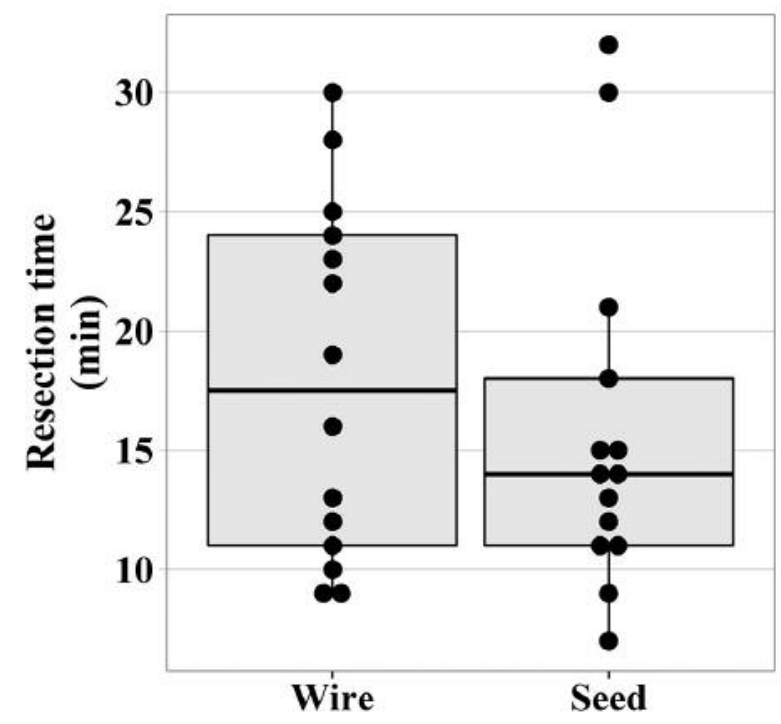

Figure 3. Boxplot together with individual data points shows the tumor resection time for both groups. (Mann-Whitney U-test: $p=0.541$ ). The boxplots illustrate the median (bold line) and $25^{\text {th }}$ and $75^{\text {th }}$ percentiles (box) of the samples. Whiskers represent the data range excluding outliers (values extending more than $1.5 \times$ interquartile range from the upper and lower box limits).

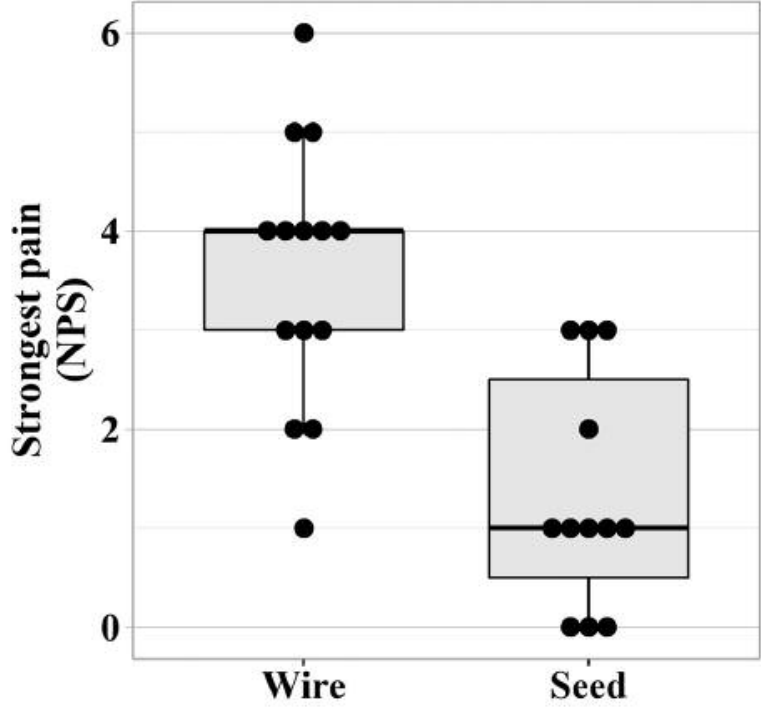

Figure 4. Boxplot together with individual data points shows the level of strongest pain on the numerical pain scale (NPS) after the localization procedure (Mann-Whitney U-test: $p<0.001)$. The boxplots illustrate the median (bold line) and $25^{\text {th }}$ and $75^{\text {th }}$ percentiles (box) of the samples. Whiskers represent the data range excluding outliers (values extending more than 1.5x interquartile range from the upper and lower box limits).

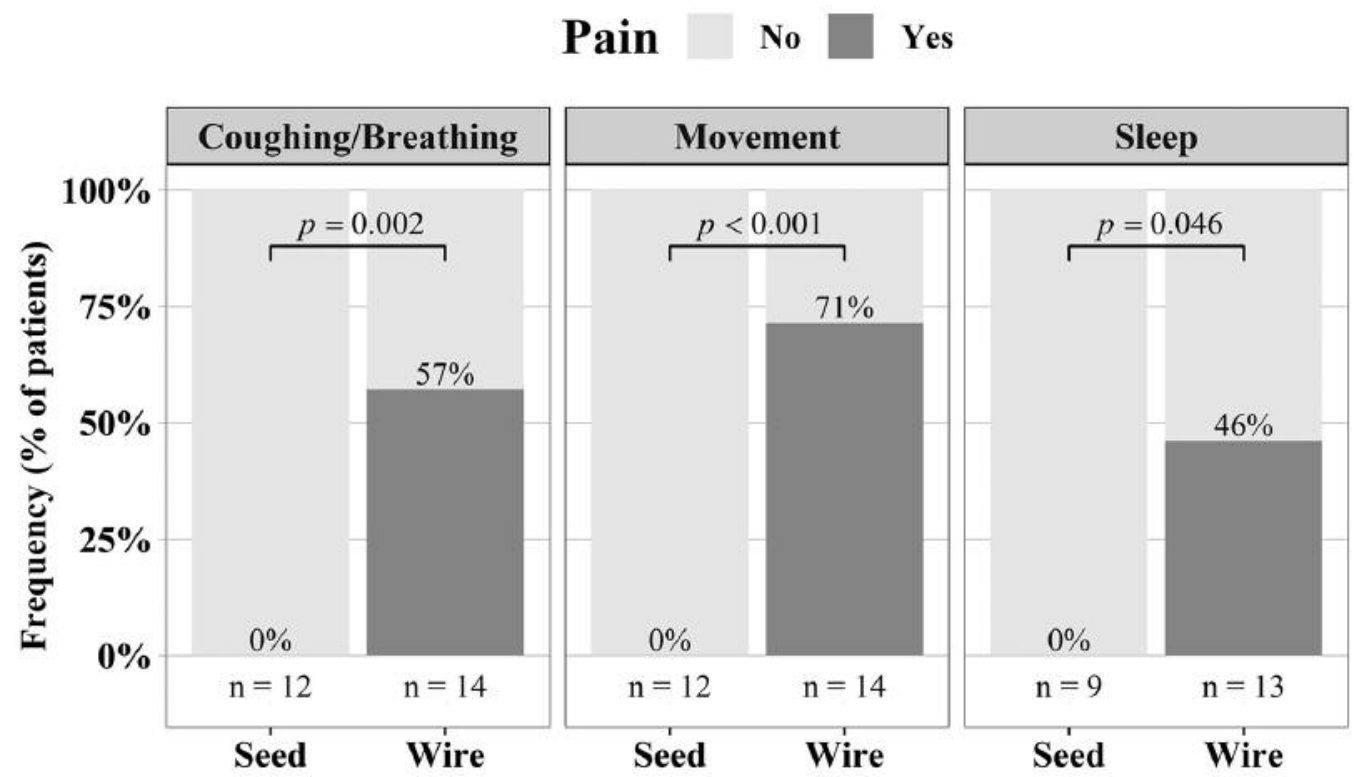

Figure 5. Bar chart comparing pain-related restriction of coughing/breathing, movement and sleep between the seed and the wire localization groups. p-Values were calculated using Fisher's exact test.

Significantly lower pain levels were reported after seed localization compared to WGL (Figure 4, $p<0.001$ ). Postinterventional pain-related restrictions are shown in Figure 5. In the seed group, significantly fewer patients (none in fact) suffered from impairment of breathing/coughing ( $0 \%$ versus $57 \%, p=0.002)$, movement $(0 \%$ versus $71 \%, p<0.001)$ or sleep $(0 \%$ versus $46 \%$, $p=0.046$ ). 


\section{Discussion}

The results of our study show that magnetic seed localization is superior to standard wire localization in regard to patient comfort. In addition, this new method showed slightly lower operative times (14 vs. $17.5 \mathrm{~min}$ ) which we expect to decrease once surgeons are more experienced with seed localization. We have shown here that patients reported higher pain levels after the localization procedure when WGL was used in comparison to Magseed ${ }^{\circledR}$ localization. Similar results have been published when WGL was compared to RSL (8). Greater convenience and patient satisfaction, as well as simplified scheduling, are advantages of RSL, which have been well reported $(8,9)$. As the insertion procedure is comparable, those advantages also apply to magnetic seeds but without the inconvenience of radiation safety requirements. Furthermore, it is currently recommended to remove radioactive seeds 5-7 days after insertion, while Magseeds ${ }^{\circledR}$ are cleared by the U. S. Food and Drug Administration for long-term and soft-tissue implantation (10, 11). This provides new possibilities in the usage of magnetic seeds, e.g. making them applicable for neoadjuvant regimes. Using magnetic seeds in labeling diseased axillary lymph nodes before the start of neoadjuvant chemotherapy will particularly have tremendous advantages for performing successful targeted axillary dissection. Successful localization of lymph nodes with magnetic seeds in the adjuvant setting has already been reported (12), so hopefully there will soon be data regarding its feasibility in the neoadjuvant setting. In addition to the use of Magseeds ${ }^{\circledR}$ in the neoadjuvant setting, it might be interesting to look at the possible differences in esthetic outcomes and satisfaction using Magseed ${ }^{\circledR}$ instead of WGL, as the surgical approach is no longer dependent on the wire's entry point.

Limitations of this study are the small number of patients, the lack of randomization and a possible selection bias regarding patients with suspicious microcalcifications. Because the latter are only seen in mammography, the localization had to be done by the Radiology Department of our Clinic, which currently does not conduct seed localizations. This is why these patients were more often allocated to the wire group. DCIS is highly associated with microcalcifications in mammography, which explains the higher rate in the wire group (13). It is also more often associated with positive resection margins, possibly leading to the rather high rate of positive margins in the wire group (14). Taking this into account, our results do not allow us to make any conclusions about resection safety. We point out that similar safety has, however, been shown in other studies (7).

\section{Conflicts of Interest}

MMK received a travel grant and speaker's fee from Sysmex (Sysmex Corporation, Kobe, Japan). The other Authors declare that they have no competing interests.

\section{Authors' Contributions}

FK, CS: Prepared the article; MMK, JUB, JG, JK: performed surgical procedures; OS: prepared the table; OS, MMK, FK, CS, IA: data collection and analysis; MMK: revised the final draft of the article.

\section{Acknowledgements}

The material and equipment were provided free of charge by Sysmex (Sysmex Corporation, Kobe, Japan). Apart from that, this research did not receive any specific grant from funding agencies in the public, commercial, or not-for-profit sectors.

\section{References}

1 Simbrich A, Wellmann I, Heidrich J, Heidinger O and Hense HW: Trends in advanced breast cancer incidence rates after implementation of a mammography screening program in a German population. Cancer Epidemiol 44: 44-51, 2016. PMID: 27470937. DOI: 10.1016/j.canep.2016.07.006

2 Nadeem R, Chagla LS, Harris O, Desmond S, Thind R, Titterrell $\mathrm{C}$ and Audisio RA: Occult breast lesions: A comparison between radioguided occult lesion localisation (ROLL) $v s$. Wire-guided lumpectomy (WGL). Breast 14(4): 283-289, 2005. PMID: 15985370. DOI: $10.1016 /$ j.breast.2005.04.002

3 Chan BK, Wiseberg-Firtell JA, Jois RH, Jensen K and Audisio RA: Localization techniques for guided surgical excision of nonpalpable breast lesions. Cochrane Database Syst Rev (12): CD009206, 2015. PMID: 26718728. DOI: 10.1002/14651858. CD009206.pub2

4 Wang GL, Tsikouras P, Zuo HQ, Huang MQ, Peng L, Bothou A, Zervoudis $\mathrm{S}$ and Tobias Teichmann A: Radioactive seed localization and wire-guided localization in breast cancer: A systematic review and meta-analysis. J BUON 24(1): 48-60, 2019. PMID: 30941951.

5 Volders JH, Haloua MH, Krekel NM, Meijer S and van den Tol PM: Current status of ultrasound-guided surgery in the treatment of breast cancer. World J Clin Oncol 7(1): 44-53, 2016. PMID: 26862490. DOI: 10.5306/wjco.v7.i1.44

6 Harvey JR, Lim Y, Murphy J, Howe M, Morris J, Goyal A and Maxwell AJ: Safety and feasibility of breast lesion localization using magnetic seeds (Magseed): A multi-centre, open-label cohort study. Breast Cancer Res Treat 169(3): 531-536, 2018. PMID: 29453521. DOI: 10.1007/s10549-018-4709-y

7 Zacharioudakis K, Down S, Bholah Z, Lee S, Khan T, Maxwell AJ, Howe $M$ and Harvey J: Is the future magnetic? Magseed localisation for non palpable breast cancer. A multi-centre non randomised control study. Eur J Surg Oncol 45(11): 2016-2021, 2019. PMID: 31288944. DOI: 10.1016/j.ejso.2019.06.035

8 Bloomquist EV, Ajkay N, Patil S, Collett AE, Frazier TG and Barrio AV: A randomized prospective comparison of patientassessed satisfaction and clinical outcomes with radioactive seed localization versus wire localization. Breast J 22(2): 151-157, 2016. PMID: 26696461. DOI: $10.1111 /$ tbj.12564

9 Murphy JO, Moo TA, King TA, Van Zee KJ, Villegas KA, Stempel M, Eaton A, St Germain JM, Morris E and Morrow M: Radioactive seed localization compared to wire localization in breast-conserving surgery: Initial 6-month experience. Ann Surg 
Oncol 20(13): 4121-4127, 2013. PMID: 23943024. DOI $10.1245 / \mathrm{s} 10434-013-3166-4$

10 Greenwood HI, Dodelzon K and Katzen JT: Impact of advancing technology on diagnosis and treatment of breast cancer. Surg Clin North Am 98(4): 703-724, 2018. PMID: 30005769 DOI: 10.1016/j.suc.2018.03.006

11 U. S. National Library of Medicine. ClinicalTrials gov Identifier: NCT03718455. Available at: https://clinicaltrials.gov/ct2/show/ NCT03718455. (Last accessed on 10th October 2019)

12 Greenwood HI, Wong JM, Mukhtar RA, Alvarado MD and Price ER: Feasibility of magnetic seeds for preoperative localization of axillary lymph nodes in breast cancer treatment. Am J Roentgenol 213(4): 953-957, 2019. PMID: 31166765. DOI: 10.2214/AJR.19.21378.
13 Poplack SP and Wells WA: Ductal carcinoma in situ of the breast: Mammographic-pathologic correlation. Am J Roentgenol 170(6): 1543-1549, 1998. PMID: 9609171. DOI: 10.2214/ajr. 170.6.9609171

14 Langhans L, Jensen M-B, Talman M-LM, Vejborg I, Kroman N and Tvedskov TF: Reoperation rates in ductal carcinoma in situ $v s$. invasive breast cancer after wire-guided breast-conserving surgery. JAMA Surg 152(4): 378-384, 2017. PMID: 28002557. DOI: $10.1001 /$ jamasurg.2016.4751

Received January 10, 2020

Revised January 26, 2020

Accepted January 29, 2020 